\title{
Motility and chemotaxis of a Xanthobacter sp. isolated from roots of rice
}

\author{
H. KeITH ReDing† and Juergen WiEgeL* \\ Department of Microbiology, University of Georgia, Athens, Georgia, USA
}

(Received 14 December 1992; accepted 4 January 1993)

\begin{abstract}
Chemotaxis and substrate-regulated motility of the rice isolate Xanthobacter sp. JW-KR2 was studied. Growth on alcohols produced motile cells possessing several peritrichous flagella while growth in the presence of organic acids, such as succinate, repressed flagellar synthesis, leading to nonmotile cells. Addition of 2.5 mM-adenosine $3^{\prime}, 5^{\prime}$-cyclic monophosphate (cAMP) to the culture resulted in motile cells even when succinate was the sole carbon source. Chemotaxis assays using microcapillary pipettes revealed a positive response to 1-propanol, 2-propanol, 1-butanol, 2-butanol and 1-pentanol but not to methanol, ethanol, isoamyl alcohol, hexanol, sugars, Casamino acids, tricarboxylic acid (TCA) cycle intermediates, butyrate, propionate, acetone or rice root exudates. The presence of TCA cycle intermediates in the chemotaxis assay inhibited chemotaxis towards butanol. Although the direct role of mobility and chemotaxis in the Xanthobacter-rice interaction is uncertain, one possibility is that Xanthobacter uses alcohols as a signal to move towards microaerobic zones, such as the rice rhizosphere, where carbon and energy sources such as $\mathrm{H}_{2}, \mathrm{CO}_{2}$, organic acids, alcohols and other anaerobic metabolites are present.
\end{abstract}

\section{Introduction}

Recently, Xanthobacter strains were isolated from the roots of wetland rice in Japan (Oyaizu-Masuchi \& Komagata, 1988) and Arkansas, USA (Reding et al., 1991). Xanthobacter sp. JW-KR1 multiplies in the rhizosphere of gnotobiotically grown rice and colonizes the root surface, resulting in a small benefit in plant top dry weight and number of leaves per rice plant after $41 \mathrm{~d}$ (Reding et al., 1991).

One important factor in the colonization of a plant rhizosphere may be bacterial motility and chemotaxis (Ashby et al., 1988; Gaworzewska \& Carlile, 1982; Mellor et al., 1987; Reinhold et al., 1985). Because rice plants exude sugars, organic acids and amino acids from their roots into the surrounding rhizosphere (Boureau, 1977), a chemical gradient is formed towards which organisms may migrate. This gradient may in turn facilitate colonization of the rhizoplane by microorganisms.

Until recently, only strains of Xanthobacter agilis were considered motile (Jenni \& Aragno, 1987). Reding et al. (1992) found that motility of Xanthobacter flavus strains 301 and H4-14 was carbon-source-dependent. Strains of

\footnotetext{
*Author for correspondence. Tel (706) 542 2651; Fax (706) 5422674.

$\dagger$ Present address: Department of Microbiology, Medical School, University of Minnesota, Minneapolis, MN 55455-0312, USA.
}

Abbreviation: TCA, tricarboxylic acid.
Xanthobacter autotrophicus are considered nonmotile (Wiegel, 1991; Wiegel \& Schlegel, 1984) although motile cells of $X$. autotrophicus 7cSF (a slime-reduced mutant of $X$. autotrophicus $7 \mathrm{c}$ ) have been observed during very early exponential phase when cultured with propanol as the sole carbon source (Reding et al., 1992). In this paper, we describe the requirements for motility and flagellation of Xanthobacter sp. JW-KR2 isolated from the roots of rice. In addition, we report on the unusual chemotaxis of Xanthobacter towards $\mathrm{C}_{3}$ to $\mathrm{C}_{5}$ aliphatic alcohols and the inhibition of chemotaxis toward butanol by the presence of TCA cycle intermediates.

\section{Methods}

Cultivation and maintenance of strains. Xanthobacter sp. JW-KR2 isolated from the roots of rice was used as a representative strain for the motility and chemotaxis experiments. Cells were cultivated routinely at $30^{\circ} \mathrm{C}$ in medium A, a defined mineral salts medium (Wiegel \& Schlegel, 1976). Medium A contains no organic carbon unless specified. Stock cultures were grown either on $80 \%(\mathrm{v} / \mathrm{v}) \mathrm{H}_{2}, 10 \%(\mathrm{v} / \mathrm{v}) \mathrm{CO}_{2}$ and $10 \%$ (v/v) $\mathrm{O}_{2}$, or $0.1 \% 1$-propanol with an air atmosphere. Cultures were either kept at $4{ }^{\circ} \mathrm{C}$ for working stocks or at $-80^{\circ} \mathrm{C}$ in $50 \%(\mathrm{v} / \mathrm{v})$ glycerol for long-term storage.

Growth rates and motility. Cells were cultured in sealed Hungate tubes containing $5 \mathrm{ml}$ medium $\mathrm{A}$ ( $\mathrm{pH} \mathrm{6.8)}$ with an appropriate carbon source $(0.1 \%, \mathrm{w} / \mathrm{v})$ and $15 \mathrm{ml}$ air in the headspace. Cultures were routinely shaken at 140 r.p.m. in a model G25 incubator/shaker (New Brunswick Scientific). The sealed tube prevented evaporation of volatile carbon sources but still provided sufficient oxygen for growth. Growth was monitored by measuring $\mathrm{OD}_{600}$. Chemicals were reagent grade (Sigma). To determine the percentage of motile cells, wet mounts 
were viewed under phase-contrast using an Olympus Vanox light microscope. Swimming rates were determined using a Zeiss phasecontrast microscope. Images were recorded in field mode with a Panasonic Super VHS cassette recorder using a Dage-MTI CCD72x camera.

Electron microscopy. Bacterial samples were negatively stained with $4 \%$ uranyl acetate using the single-carbon-layer technique of Valentine et al. (1968). Samples were examined using a JEOL 100CXII electron microscope at $80 \mathrm{keV}$.

Chemotaxis assays. Assays were conducted using microcapillary pipettes (Currier \& Strobel, 1976). To produce motile, radiolabelled cells, Xanthobacter sp. JW-KR2 was grown on Tryptic Soy Agar (Sigma) at $30^{\circ} \mathrm{C}$ for $3 \mathrm{~d}$ before being transferred into medium $\mathrm{A}$ containing $0.2 \%$ succinate as the carbon source and $100 \mu \mathrm{Ci}(3.7 \mathrm{MBq})$ [trans ${ }^{35}$ S]methionine $\quad\left(1117 \mathrm{Ci} \mathrm{mmol}^{-1} \quad\left(41.33 \mathrm{MBq} \mathrm{mmol}^{-1}\right)\right.$; ICN Biomedicals). At mid-exponential phase (around $1 \times 10^{8}$ cells ml $^{-1}$ ), the cells were centrifuged at $4000 \mathrm{~g}$ for $6 \mathrm{~min}$ under sterile conditions, then the pellet was washed and resuspended in an equal volume of medium A lacking a carbon source. This routinely caused $50-80 \%$ of the cells to become motile within $3 \mathrm{~h}$. A $25 \mu \mathrm{l}$ aliquot of the motile bacterial suspension was dispensed into an Eppendorf vial ( $0.5 \mathrm{ml}$ capacity). A $5 \mu \mathrm{l}$ microcapillary pipette was filled with the test compound dissolved in medium A, then the top of the pipette was heat-sealed and allowed to cool at room temperature. A single pipette containing the test compound in medium $\mathrm{A}$ or medium $\mathrm{A}$ alone was inserted into the bacterial suspension. After $90 \mathrm{~min}$ at $30^{\circ} \mathrm{C}$, the microcapillary pipette was removed, the exterior was rinsed with distilled water, and the contents were emptied into a scintillation vial containing Scintiverse BD cocktail (Fisher Scientific). Chemotaxis was quantified by counting each sample for 2 min with a Beckman model LS 5801 scintillation counter. The relative chemotactic response is expressed as the number of bacteria entering the microcapillary pipette containing the test compound divided by the number of bacteria randomly entering the control microcapillary pipette which contained medium A lacking any carbon source. Responses for three to five replicate samples were determined statistically using Duncan's Multiple Range Test $(P=$ 0.05).

To test the ability of various carbon sources to inhibit chemotaxis of Xanthobacter JW-KR2, the test carbon source from a concentrated stock solution was added to a $25 \mu \mathrm{l}$ motile cell suspension. Then, a microcapillary pipette containing $150 \mathrm{~mm}$-butanol plus the test compound was inserted. After $90 \mathrm{~min}$, the microcapillary tube was removed and processed as described above.

To collect rice root exudates aseptically, ten plants were cultivated in gnotobiotic assembly as described previously (Reding et al., 1991). After $10 \mathrm{~d}$, the plant growth medium $(50 \mathrm{ml})$ was removed, lyophilized, and resuspended in $1 \mathrm{ml}$ sterile distilled water. Glucose, fructose and sucrose concentrations were determined using a Combination Test Kit (Boehringer Mannheim).

\section{Results and Discussion}

\section{Effect of carbon source and adenosine-3', $5^{\prime}$-cyclic monophosphate (cAMP) on motility}

Xanthobacter sp. JW-KR2 utilizes a wide range of carbon sources, including organic acids, amino acids, alcohols and some sugars (Reding et al., 1991). Growth in medium containing methanol, ethanol, 1-propanol, 2propanol, or butanol as the sole carbon source resulted in motile cells possessing two to five peritrichous flagella with a type I fine structure similar to $X$. agilis (Fig. 1;
Aragno et al., 1977). Growth in medium containing citrate, fumarate, glutamate, glutamine, 2-oxoglutarate or succinate resulted in nonmotile, nonflagellated cells (data not shown). Other Xanthobacter strains isolated from rice (JW-KR1 to JW-KR6) behaved similarly. Xanthobacter sp. JW-KR2 utilized succinate with a doubling time of $3.5 \mathrm{~h}$ during mid-exponential growth phase (Fig. 2). These cells were nonmotile and lacked flagella as determined by electron microscopy (data not

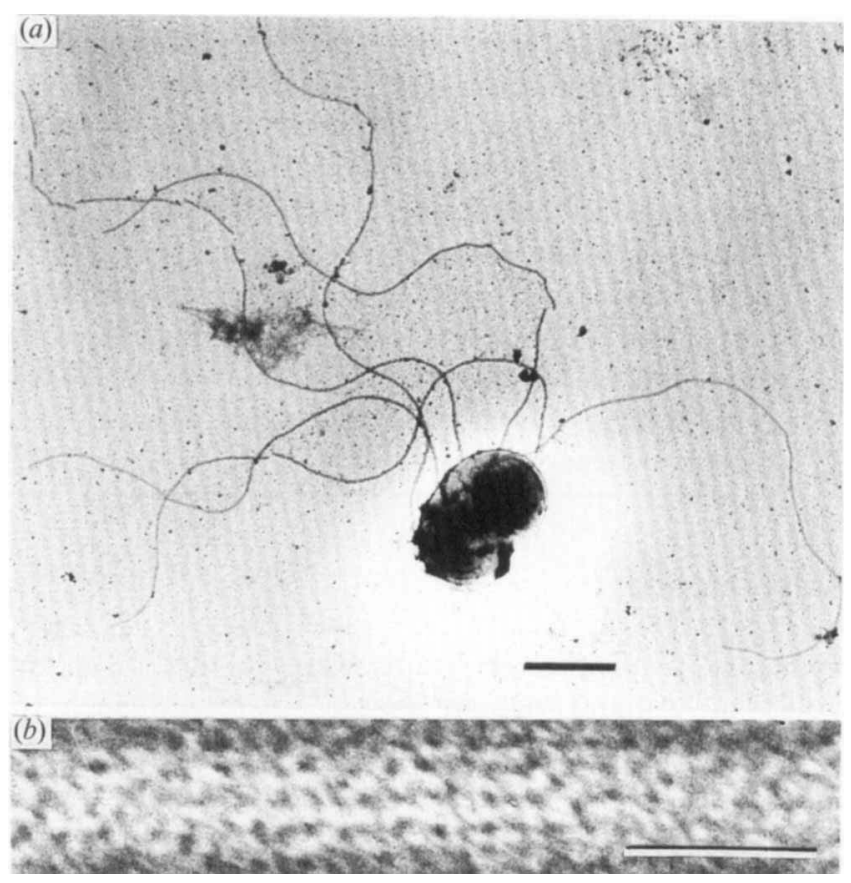

Fig. 1. (a) Electron micrograph of a negatively-stained sample of Xanthobacter sp. JW-KR2 having several peritrichous flagella. Bar, $1 \mu \mathrm{m}$. (b) Type I flagellar fine structure similar to that of $X$. agilis (Aragno et al., 1977). Bar, $20 \mathrm{~nm}$.

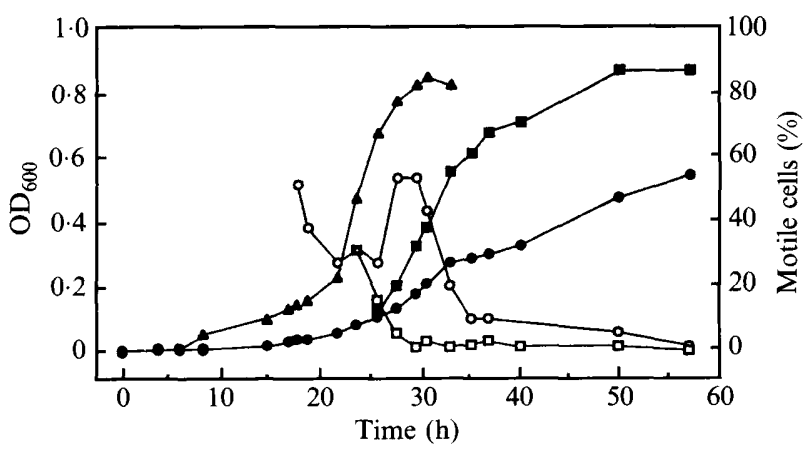

Fig. 2. Growth (filled symbols) and percentage of motile cells (open symbols) during growth on $0.1 \%$ succinate $(\boldsymbol{\Lambda}), 0 \cdot 1 \%$ 1-propanol $(\boldsymbol{\bullet}$, $\bigcirc$ ), and $0.1 \%$ propanol plus $0.1 \%$ succinate $(\boldsymbol{\square}, \square$; succinate was added to the propanol culture at $25 \mathrm{~h}$ after inoculation). Values represent the mean of 3-6 replicates. 


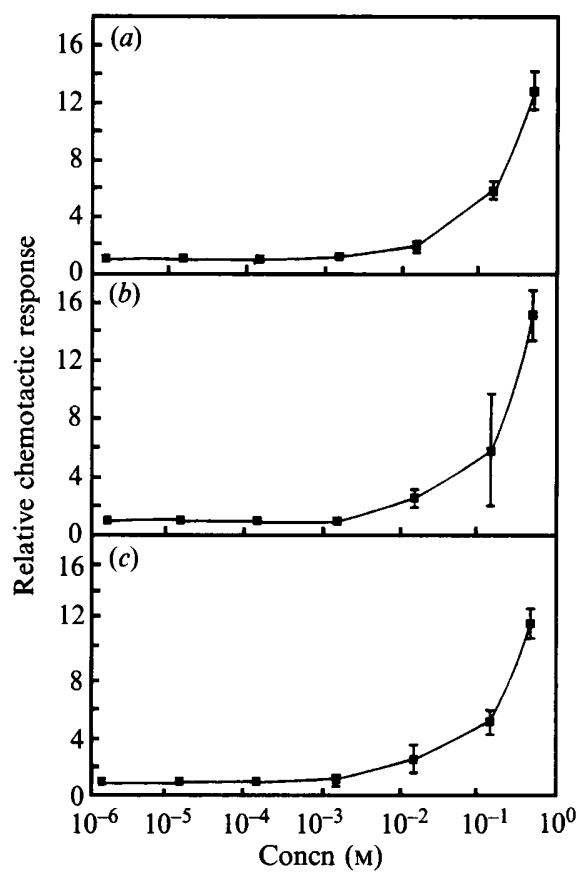

Fig. 3. Relative chemotactic response (response relative to control lacking attractant) to various concentrations of $(a)$ 1-propanol, $(b) 2$ propanol, or (c) 1-butanol. Values are the mean of 4 replicates \pm SD.

shown). Upon transfer of the culture to medium containing 1-propanol as the sole carbon source $50 \%$ of the cells became mobile with a slower doubling time of $5.5 \mathrm{~h}$ at mid-exponential growth phase (Fig. 2). When succinate was added to the cultures growing on propanol at $25 \mathrm{~h}$ after inoculation, the growth rate increased and the percentage of motile cells decreased to less than $1 \%$ within $6 \mathrm{~h}$. The reduction of motile cells with the addition of succinate is likely to be due to repression of flagella synthesis. Motility of Xanthobacter sp. JW-KR2 is apparently induced by lack of quickly utilizable carbon in the medium. The downshift from succinate to 1propanol as the carbon source resulted in a slower growth rate and synthesis of flagella. Flagella were also synthesized when cells grown in medium containing succinate or fumarate were transferred to medium lacking carbon. These cells became motile within 2 to $3 \mathrm{~h}$ of removal of the carbon source and remained motile for up to $32 \mathrm{~h}$ (data not shown). Xanthobacter spp. produce large amounts of poly- $\beta$-hydroxybutyrate as carbon storage, which can account for as much as $70 \%$ of the cellular dry weight (Wiegel \& Schlegel, 1984). Presumably, Xanthobacter spp. are able to utilize the poly$\beta$-hydroxybutyrate to synthesize flagella and become motile under carbon starvation.

In cultures of Xanthobacter sp. JW-KR2 grown on $0 \cdot 1 \%$ succinate, the addition of $2 \cdot 5 \mathrm{mM}$-cAMP produced motile cells with a slower growth rate, comparable to cells grown on 1-propanol alone (for typical growth curves of succinate or propanol cultures, see Fig. 2). Growth and motility of cells cultured on $0.1 \% 1$ propanol alone were not affected by the addition of 2.5 mM-cAMP. In this respect, the effect of cAMP on Xanthobacter sp. JW-KR2 is comparable to the effects observed with Escherichia coli. Flagellation of E. coli, and thus motility, is also carbon-source-dependent. In this case, the presence of glucose represses synthesis of flagella (Adler \& Templeton, 1967). Addition of $2.5 \mathrm{~mm}-$ cAMP to medium containing glucose produces motile cells (Dobrogosz \& Hamilton, 1971) with a slower growth rate (Mikheeva, 1985). The presence of cAMP allows the flagellar operon to be transcribed even in medium containing glucose (Silverman \& Simon, 1974; Yokota \& Gots, 1970). Surprisingly, the addition of cAMP to Xanthobacter $\mathrm{sp}$. JW-KR2 cultures containing both succinate and 1-propanol neither decreased the growth rate nor resulted in the cells becoming motile (data not shown). The reason for this unexpected occurrence is unclear. X. flavus 301 behaved similarly with respect to cAMP and motility (data not shown).

\section{Chemotaxis}

Xanthobacter sp. JW-KR2 demonstrated unequivocal chemotaxis toward 1-propanol, 2-propanol and butanol between $15 \mathrm{~mm}$ and $500 \mathrm{~mm}$ (nominal concentrations in the capillary tubes; see below) with no peak response observed (Fig. 3). The lack of a peak response is rather atypical for a dose-response curve; however, control experiments using nonmotile cells did not mimic a positive response when up to $500 \mathrm{~mm}$-alcohol was present in the microcapillary tube. This rules out false positive results due to density-dependent hydrodynamic effects which can occur if high concentrations are present in the microcapillary tube ( Shonnard et al., 1992). No response was detected to the alcohols between $1.5 \mathrm{~mm}$ and $0.0015 \mathrm{~mm}$. Unequivocally positive responses (relative responses of $3.4,3.6$ and 8.9 , respectively) were observed for $150 \mathrm{~mm}$-pentanol, $15 \mathrm{~mm}$-pentanol and $150 \mathrm{~mm}-2$ butanol, but no positive responses were detected below these concentrations (down to $0.0015 \mathrm{~mm}$; data not shown). The likely oxidation products of 1-propanol, 2propanol and butanol (propionate, acetone and butyrate, respectively) did not produce a positive chemotactic response, indicating that the chemotaxis is specific for the alcohols. Xanthobacter sp. JW-KR2 showed no significant chemotaxis towards glucose, fructose, sucrose, methanol, ethanol, isoamyl alcohol, hexanol, citrate, fumarate, oxalate, 2-oxoglutarate, malate or succinate at concentrations between 0.0015 and $150 \mathrm{mM}$. Furthermore, no response was detected toward concentrated rice root exudates (glucose, fructose and sucrose concentrations of $4.4,1 \cdot 1$, and $0.23 \mathrm{~mm}$, respectively). To our 
Table 1. Substrate inhibition of chemotaxis to $150 \mathrm{mM}$-butanol

Values represent the percentage $( \pm \mathrm{SD})$ of cells entering the capillary tube containing $150 \mathrm{mM}$ butanol plus the test inhibitor relative to the control (150 mM-butanol only). The cell suspension also contained a similar amount of the test compound so as not to create a second gradient.

\begin{tabular}{lccccc}
\hline \hline & \multicolumn{5}{c}{ Concn (mM) } \\
\cline { 2 - 6 } Inhibitor & 0 & $1 \cdot 5$ & $7 \cdot 5$ & 15 & 75 \\
\hline Citrate & $100 \pm 16$ & $183 \pm 73$ & $125 \pm 41$ & $96 \pm 12$ & $17 \pm 6^{*}$ \\
Fumarate & $100 \pm 16$ & $74 \pm 31^{*}$ & $68 \pm 38^{*}$ & $40 \pm 4^{*}$ & $24 \pm 4^{*}$ \\
Succinate & $100 \pm 16$ & $14 \pm 5^{*}$ & $28 \pm 9^{*}$ & $43 \pm 4^{*}$ & $16 \pm 4^{*}$ \\
Glutamate & $100 \pm 16$ & $85 \pm 15$ & $10 \pm 4^{*}$ & $13 \pm 4^{*}$ & $20 \pm 8^{*}$ \\
Glucose & $100 \pm 16$ & $88 \pm 15$ & $86 \pm 32$ & $93 \pm 14$ & $105 \pm 20$ \\
Sucrose & $100 \pm 16$ & $172 \pm 30$ & $112 \pm 59$ & $179 \pm 24$ & $120 \pm 5$ \\
\hline \hline
\end{tabular}

${ }^{*}$ A statistically significant decrease in response compared to the control as determined by Duncan's Multiple Range Test.

$\dagger$ Strain JW-KR2 does not utilize sucrose.

knowledge, this is the first report of bacterial chemotaxis towards aliphatic alcohols, although chemotaxis to aromatic alcohols (Ashby et al., 1988) and sugar alcohols (Haneline et al., 1991) has been reported. Other studies have reported negative chemotaxis, i.e. bacteria move away from alcohols (Tso \& Adler, 1974; Smith \& Doetsch, 1969). The only report of chemotaxis towards aliphatic alcohols is for zoospores of the phytopathogenic fungus, Phytophthora cinnamomi, which are chemotactic towards methanol, ethanol, n-propanol and nbutanol (Allen \& Newhook, 1973). Ethanol is produced by some water-stressed plants (Bolton \& Erickson, 1970). Therefore, Allen \& Newhook (1973) postulated that ethanol produced by plant roots may encourage the accumulation of zoospores at the site of exudation.

Glucose is the major carbon source found in root exudates of rice, accounting for $62 \%$ of the total carbon quantified (Boureau, 1977). Organic acids, such as oxalic acid, succinate, aconitate and citrate, are found only in minor amounts. It is not known whether aliphatic alcohols are exuded from the roots of rice under field conditions. Root exudates are routinely analysed after lyophilization, thus any alcohol present would be lost in this process.

The chemotactic response toward aliphatic alcohols may have no direct function in attracting Xanthobacter spp. to the roots, but alcohols may serve as a signal for Xanthobacter spp. to move towards microaerobic environments containing anaerobic metabolites such as $\mathrm{H}_{2}, \mathrm{CO}_{2}$, organic acids and alcohols. These anaerobic fermentation products are the main carbon and energy sources for Xanthobacter spp. Most Xanthobacter spp. are very limited with respect to sugar utilization: some strains use no sugars, whereas some can use fructose and/or sucrose and mannose. The utilization of glucose requires a long adaptation period for most strains, including JW-KR2 (Wiegel, 1991). Adler (1975) and Reinhold et al. (1985) reported chemotaxis to compounds which are not utilized for growth, suggesting that this may serve to place the organism in a nutritious environment. For Xanthobacter spp. aerotaxis to low $\mathrm{pO}_{2}$ might accomplish the same goal; however, no banding of Xanthobacter sp. JW-KR2 cells was observed in wet mounts under cover slips, which indicates an absence of a strong aerotactic behaviour.

The presence of TCA cycle intermediates or glutamate in the cell suspension and microcapillary pipette inhibited the positive chemotactic response to butanol, whereas the non-utilizable substrate sucrose enhanced the response (Table 1). Although growth in the presence of these compounds eventually leads to repression of flagellar synthesis, microscopic examination of the cell suspension at the end of the 90 min chemotaxis assay revealed no decrease in the percentage of motile cells or the rate of swimming, which was $19 \pm 5 \mu \mathrm{m} \mathrm{s}^{-1}$ (8-18 body lengths $\mathrm{s}^{-1}$ ) and $17 \pm 4 \mu \mathrm{m} \mathrm{s}^{-1}$ in the absence and presence of $7.5 \mathrm{~mm}$-succinate, respectively. Therefore, the inhibition must be due to a reduction of the chemotactic response towards butanol. The presence of glucose or sucrose, which are not readily utilized as carbon sources, did not inhibit chemotaxis to butanol.

A similar inhibitory effects on chemotaxis was observed with cultures of Vibrio furnissii (Bassler et al., 1991), where the presence of TCA cycle intermediates or compounds that feed into the TCA cycle inhibited chemotaxis to sugars and glutamine. Moreover, the degree of inhibition correlated with the oxidation rate of each TCA cycle intermediate. Bassler et al. (1991) suggested a link between catabolism and taxis in $V$. furnissii which may involve an interaction between the two systems. Our results suggest that a similar system may exist for Xanthobacter sp. JW-KR2. 
Because Xanthobacter sp. JW-KR2 did not show chemotaxis toward lyophilized rice root exudates or pure compounds reported to be produced by the roots, the role of motility and chemotaxis in the Xanthobacter-rice association is uncertain. Alcohols to which this Xanthobacter is attracted are likely to occur in anaerobic rice paddy soils; however, to our knowledge, alcohol concentrations in the rhizosphere compared to the nonrhizosphere soil have not been investigated. Chemotaxis to alcohol concentrations above $100 \mathrm{~mm}$ are unlikely to be of biological significance since they are not expected to occur at this concentration in soil and sediments. However, an unambiguous positive chemotactic response was obtained at concentrations as low as $15 \mathrm{~mm}$. Hazelbauer \& Adler (1971) found that the concentration of solute in a capillary tube to which $E$. coli can respond in a chemotaxis assay is 200 -fold lower than the actual concentration of the solute in the capillary tube. This is due to the design of the assay, in which diffusion out of the capillary tube forms a concentration gradient. Therefore, alcohol concentrations in the soil as low as $0.075 \mathrm{~mm}(15 \mathrm{~mm}$ in the assay tube) might be able to elicit a chemotactic response by Xanthobacter sp. JW-KR2. Concentrations of $0.1 \mathrm{~mm}-$ aliphatic alcohols are of biological significance. Such (and higher) concentrations have been found in sediment samples from lakes in the vicinity of Athens, GA, USA (unpublished results). Thus, we believe that the observed chemotactic response, despite the relative high concentrations required for an unambiguous response in the test assay used, is of biological significance.

Overall, the data imply that under carbon-limiting conditions (i.e. absence of quickly utilizable carbon and energy sources) chemotaxis to aliphatic alcohols may direct Xanthobacter spp. to zones in the soil where products of anaerobic metabolism are likely to be present. Once reaching that point, utilization of organic acids (TCA cycle intermediates) would inhibit further chemotaxis to alcohols and eventually repress flagellar synthesis, leading to nonmotile cells.

This research was funded by a Biotechnology grant from the Office of the Vice President for Research, the University of Georgia and in part by a grant to J. W. from the US Department of Energy (DE-FG0986-ER-13614). We thank Drs Palmer Rogers and Stewart Goldstein for allowing additional experiments to be performed in their laboratory.

\section{References}

Adler, J. (1975). Chemotaxis in bacteria. Annual Review of Biochemistry 44, 341-356.

AdleR, J. \& Templeton, B. (1967). The effect of environmental conditions on the motility of Escherichia coli. Journal of General Microbiology 46, 175-184.

Allen, R. N. \& Newhook, F. J. (1973). Chemotaxis of zoospores of Phytophthora cinnamomi to ethanol in capillaries of soil pore dimensions. Transactions of the British Mycological Society 61, 287-302.

Aragno, M., Walther-Mauruschat, A., Mayer, F. \& Schlegel, H. G. (1977). Micromorphology of Gram-negative hydrogen bacteria. I. Cell morphology and flagellation. Archives of Microbiology 114, 93-100.

Ashby, A. M., Watson, M. D., Loake, G. J. \& Shaw, C. H. (1988). Ti plasmid-specific chemotaxis of Agrobacterium tumefaciens $\mathrm{C} 58 \mathrm{Cl}$ toward vir-inducing phenolic compounds and soluble factors from monocotyledonous and dicotyledonous plants. Journal of Bacteriology 170, 4181-4187.

Bassler, B. L., Gibbons, P. J., Yu, C. \& Roseman, S. (1991). Chitin utilization by marine bacteria. Journal of Biological Chemistry 266, 24268-24275.

Bolton, E. F. \& ERICKSON, A. E. (1970). Ethanol concentration in tomato plants during soil flooding. Agronomy Journal 62, 220-224.

BourEaU, M. (1977). Application de la chromatographie en phase gazeuse à l'étude de l'exsudation racinaire du riz. Cahiers ORSTROM Serie Biologie 12, 75-81.

Currier, W. W. \& Strobel, G. A. (1976). Chemotaxis of Rhizobium spp. to plant root exudates. Plant Physiology 57, 820-823.

Dobrogosz, W. J. \& Hamilton, P. B. (1971). The role of cyclic AMP in chemotaxis in Escherichia coli. Biochemistry and Biophysics Communication 42, 202-207.

Gaworzewska, E. T. \& Carlile, M. J. (1982). Positive chemotaxis of Rhizobium leguminosarum and other bacteria towards root exudates from legumes and other plants. Journal of General Microbiology 128, 1179-1188.

Haneline, S., Connelly, C. J. \& Melton, T. (1991). Chemotactic behavior of Azotobacter vinelandii. Applied and Environmental Microbiology 57, 825-829.

Hazelbauer, G. L. \& AdLer, J. (1971). Role of the galactose binding protein in chemotaxis of Escherichia coli toward galactose. Nature New Biology 230, 101-104.

JenNi, B. \& AraGno, M. (1987). Xanthobacter agilis sp. nov., a motile, dinitrogen-fixing, hydrogen-oxidizing bacterium. Systematic and Applied Microbiology 9, 254-257.

Mellor, H. Y., GlenN, A. R., Arwas, R. \& Dilworth, M. J. (1987). Symbiotic and competitive properties of motility mutants of Rhizobium trifolii 1. Archives of Microbiology 148, 34-39.

Mikheeva, G. A. (1985). Effect of cyclic 3'-5'-adenosine monophosphate on the growth rate of Escherichia coli. Microbiology 54, 704-707.

Oyaizu-Masuchi, Y. \& Komagata, K. (1988). Isolation of free-living nitrogen fixing bacteria from the rhizosphere of rice. Journal of General and Applied Microbiology 34, 127-164.

Reding, H. K., Hartel, P. G. \& Wiegel, J. (1991). Effect of Xanthobacter, isolated and characterized from rice roots, on growth of wetland rice. Plant and Soil 138, 221-229.

Reding, H. K., Croes, C. L. M., Dijkhuizen, L. \& Wiegel, J. (1992). Emendation of Xanthobacter flavus as a motile species. International Journal of Systematic Bacteriology 42, 309-311.

Reinhold, B., HuReK, T. \& FendriK, I. (1985). Strain-specific chemotaxis of Azospirillum spp. Journal of Bacteriology 162, 190-195.

Shonnard, D. R., Taylor, R. T., Tompson, A. \& KnapP, R. B. (1992). Hydrodynamic effects on microcapillary motility and chemotaxis assays of Methylosinus trichosporium OB3b. Applied and Environmental Microbiology 58, 2737-2743.

Silverman, M. \& Simon, M. (1974). Characterization of Escherichia coli flagellar mutants that are sensitive to catabolite repression. Journal of Bacteriology 120, 1196-1203.

Sмiтh, J. L. \& DoeTsCH, F. N. (1969). Studies on negative chemotaxis and the survival value of motility in Pseudomonas fluorescens. Journal of General Microbiology 55, 379-391.

Tso, W. \& ADlER, J. (1974). Negative chemotaxis in Escherichia coli. Journal of Bacteriology 118, 560-576.

Valentine, R. C., Shapiro, B. M. \& Stadtmen, E. R., (1968). Regulation of glutamine synthetase. XII. Electron microscopy of the enzyme from Escherichia coli. Biochemistry 7, 2143-2152.

WIEGEL, J. (1991). Genus Xanthobacter. In The Prokaryotes. A Handbook on the Biology of Bacteria. Ecophysiology, Isolation, 
Identification, Application, 2nd edn, vol. III, pp. 2362-2383. Edited by $\mathrm{H}$. Balows and others. New York, Amsterdam: Springer Verlag. WiEgEL, J. \& SCHLEGEL, H. G. (1976). Enrichment and isolation of nitrogen-fixing hydrogen bacteria. Archives of Microbiology 107, $139-142$.

Wiegel, J. K. W. \& Schlegel, H. G. (1984). Genus Xanthobacter
Wiegel, Wilke Baumgarten, Opitz and Schlegel 1978, 573 ${ }^{\mathrm{AL}}$. In Bergey's Manual of Systematic Bacteriology, vol. 1, pp. 325-333. Edited by N. R. Krieg \& J. G. Holt. Baltimore: Williams \& Wilkins. YокоTA, T. \& GoTs, J. S. (1970). Requirement of adenosine 3',5'-cyclic phosphate for flagella formation in Escherichia coli and Salmonella typhimurium. Journal of Bacteriology 103, 513-516. 